3. The asymptotic series. For practical purposes, it is however advantageous to give up the convergence of the expansion and turn it into an ordinary divergent asymptotic series with simpler terms to be used for $z \gg 1$. To that end, one puts $\sinh z \sim \cosh z$ $\sim e^{z} / 2$, neglects the second term between the brackets in $(2,4)$, inserts the formulae given for $I_{s+1 / 2}(z) \operatorname{nt} \leadsto(2,4)$ and rearranges the terms with respect to decreasing powers of $z$. Then one obtains

$f(z) \sim e^{z}(\pi z)^{-1 / 2}\left(1+\frac{3}{4} z^{-1}+\frac{41}{32} z^{-2}+\frac{445}{128} z^{-3}+\frac{26571}{2048} z^{\prime}+\frac{505029}{8192} z^{-5} \ldots\right)$.

The given terms yield e.g. $f(20) \sim 1.42507 \cdot 10^{7}$; actually $f(20)=1.42508 \cdot 10^{7}$.

A note on my paper

\title{
A GENERAL SOLUTION FOR THE RECTANGULAR AIRFOIL IN SUPERSONIC FLOW
}

\author{
Quarterly of Applied Mathematics, XI, 1-8 (1953) \\ By JUHN W MILFS (Iniversit!y of California)
}

It appears that the application of the Lorentz transformation to unsteady flow problems was suggested previously by P. A. Lagerstrom (unpublished lectures at California Institute of Technology, Pasadena, 1948) and by C. Gardner in his paper Time-dependant linearized supersonic flow past planar wings, Comm. on Pure and Appl. Math. 3, 33-38 (1950).

\section{BOOK REVIEWS}

Electronic analog computers. By G. A. Korn and T. M Korn. Me( (rraw-Hill Book ('ompany, Inc., New York, Toronto, London, $\mathrm{xv}+378$ pp. \$7.00

This book is both a text and an engineering reference book on the subject of d.e. electronic analog computors. The type of computer under consideration is that employed bi many commercial and government institutions. These computers are used in the solution of such problems as are encountered in the design of feedback control systems and in the development of new aircraft configurations.

In the book, the problems associated with the use of computers are first discussed. Examples are given of setup procedures and applications to show both the limitations and the versatility of these devices. Following this, the principal engineering design considerations associated with the romputer components are discussed. The d.c. amplifiers employed are analyzed at length as are the various means of obtaining special functional representations. The book is concluded with a description of several of the commercially available computers.

As a text on electronic analog computers, it is unfortunate that the a.c computer are not considered as well as the d.c. computers. Because of this the book is not sufficiently halanced for " text on analog 'omputers and would have to be supplemented by additional reading. The principal value of the book is for the orientation of mathematicians and others concerned with the solution of dynamic problems on analog computers that have either been specially designed or commercially procured 